\title{
ANÁLISE DA PRODUÇÃO, IMPORTAÇÃO, EXPORTAÇÃO E CONSUMO APARENTE DE PAPEL NO BRASIL ENTRE $1961 \mathrm{E}$ 2016
}

\author{
PRODUCTION, IMPORT, EXPORT AND APPARENT CONSUMPTION OF PAPER \\ IN BRAZIL DURING 1961-2016
}

\author{
Carlos Roberto Sanquetta ${ }^{1}$; Thiago Wendling Gonçalves de Oliveira ${ }^{2}$; \\ Ana Paula Dalla Corte ${ }^{3}$; Mateus Niroh Inoue Sanquetta ${ }^{4}$ Greyce Charllyne Benedet Maas ${ }^{5}$ \\ 1, 2, 3, 4, 5 Universidade Federal do Paraná, Curitiba, Paraná, Brasil - carlossanquetta@gmail.com; \\ thiagowendling@yahoo.com.br; anapaulacorte@gmail.com; mateus.sanquetta@gmail.com \& \\ greyce.maas@gmail.com
}

\section{RESUMO}

O segmento de papel e celulose tem posição de destaque na economia do setor florestal brasileiro e mundial. O objetivo deste estudo foi analisar a dinâmica da produção, importação, exportação e consumo aparente de três tipos de papéis ( $\mathrm{P}+\mathrm{W}$ : imprimir e escrever, $\mathrm{H}+\mathrm{S}$ : uso doméstico e sanitário e $\mathrm{N}$ : jornal) no período de 1961 a 2016. Para essa análise foram utilizados dados extraídos do sistema FAOSTAT da FAO. Os resultados encontrados demonstram que nesse período foram produzidas $77 \mathrm{Mt}$ de $\mathrm{P}+\mathrm{W}, 24 \mathrm{M}$ t de $\mathrm{H}+\mathrm{S}$ e $9 \mathrm{M}$ t de $\mathrm{N}$. As importações totalizaram $10 \mathrm{M} \mathrm{t}, 188$ mil t e $12 \mathrm{M} \mathrm{t}$, respectivamente para $\mathrm{P}+\mathrm{W}, \mathrm{H}+\mathrm{S}$ e $\mathrm{N}$. As exportações no período foram de $25 \mathrm{M} \mathrm{t}, 500 \mathrm{mil}$ t e 334 mil t, respectivamente. O consumo aparente foi de $61 \mathrm{M} \mathrm{t}, 24 \mathrm{M}$ t e $21 \mathrm{M} \mathrm{t}$, respectivamente. $\mathrm{O}$ consumo médio per capita desses papéis no período foi de 6,$98 ; 2,58$ e 2,57 $\mathrm{kg}$.hab ${ }^{-1}$. O consumo de $\mathrm{H}+\mathrm{S}$ deu-se de forma crescente em toda a série temporal, enquanto que para $\mathrm{P}+\mathrm{W}$ e $\mathrm{N}$ foi decrescente, sobretudo a partir de 2010. O Brasil é superavitário em $\mathrm{P}+\mathrm{W}$ e H+S e deficitário na balança comercial em N. Conclui-se que há uma tendência de queda de consumo em $\mathrm{P}+\mathrm{W}$ e $\mathrm{N}$ e aumento de $\mathrm{H}+\mathrm{S}$, essa tendência é verificada na produção, importação e consumo. As exportações de $\mathrm{H}+\mathrm{S}$ e $\mathrm{N}$ ainda são pouco expressivas. Já as importações de P+W e N apresentaram tendência de queda desde 2010.

PALAVRAS-CHAVE: Indústria de Papel e Celulose, Mercado de Produtos Florestais, Setor Florestal Brasileiro.

\section{ABSTRACT}

The paper and pulp sector has a remarkable position in the Brazilian and world forest economy. The temporal dynamics of the production, import, export and apparent consumption of three types of paper $(P+W$ : print and write, $\mathrm{H}+\mathrm{S}$ : household and sanitary use and $\mathrm{N}$ : newspaper) were analyzed from 1961 to 2016. Data came from FAO's FAOSTAT system. Imports totaled $10 \mathrm{M} \mathrm{t}, 188$ thousand $\mathrm{t}$ and $12 \mathrm{M} \mathrm{t}$, respectively for $\mathrm{P}+\mathrm{W}, \mathrm{H}+$ $\mathrm{S}$ and $\mathrm{N}$. Exports in the period were $25 \mathrm{M} \mathrm{t}, 500 \mathrm{t}$ and 334 thousand $\mathrm{t}$, respectively for $\mathrm{P}+\mathrm{W}, \mathrm{H}+\mathrm{S}$ and $\mathrm{N}$. The apparent consumption was $61 \mathrm{M} \mathrm{t}, 24 \mathrm{M} \mathrm{t}$ and $21 \mathrm{M} \mathrm{t}$, respectively. The average per capita consumption of these papers in the period is $6.98 ; 2.58$ and $2.57 \mathrm{~kg}$.hab-1. The consumption of $\mathrm{H}+\mathrm{S}$ increased throughout the time series, while $P+W$ and $N$ decreased, especially from 2010. Brazil has a surplus in $P+W$ and $H+S$ and a deficit in the trade balance in $\mathrm{N}$. It concludes that there is a downward trend in consumption in $\mathrm{P}+\mathrm{W}$ and $\mathrm{N}$ and increase in $\mathrm{H}+\mathrm{S}$ increase, this tendency is verified in production, import and consumption. Exports of $\mathrm{H}+\mathrm{S}$ and $\mathrm{N}$ are still not very expressive. Imports of $\mathrm{P}+\mathrm{W}$ and $\mathrm{N}$ have been falling steadily since 2010 and increasing in relation to $\mathrm{H}+\mathrm{S}$.

KEYWORDS: Pulp and Paper Industry, Forest Product Market, Brazilian Forest Sector. 


\section{INTRODUÇÃO}

O Brasil apresenta grande competitividade no mercado interno e externo de produtos florestais devido às suas características edafoclimáticas e, principalmente, pelo desenvolvimento tecnológico alcançado nas últimas décadas na silvicultura e no manejo dos povoamentos, além da industrialização dos seus produtos (MOREIRA e OLIVEIRA, 2017).

O setor de florestas plantadas, em particular, contribui com 1,1\% do PIB nacional e 6,1\% do PIB industrial, com receita bruta de $\mathrm{R} \$ 73,8$ milhões.ano-1 e saldo na balança comercial de US\$ 9,0 bilhões em 2018. O mesmo setor teve uma arrecadação de $\mathrm{R} \$ 11,5$ bilhões em tributos, o que corresponde a 0,9\% em nível nacional, gerando 3,7 milhões de empregos, sendo 508 mil diretos (IBÁ, 2018).

O investimento do setor em 2018 foi cerca de R\$ 6,5 bilhões, sendo 3,2 bilhões no manejo das florestas e 3,5 bilhões no parque fabril. A produção industrial alcançou 19,5 milhões $t$ de celulose, 10,5 milhões $t$ de papel, 7,9 milhões $t$ de painéis de madeira, 11 milhões $t$ de pisos laminados e 4,5 milhões t de carvão vegetal (IBÁ, 2018).

$A$ indústria brasileira de celulose apresentou grande crescimento nas últimas décadas. Esse movimento foi acompanhado pela instalação de grandes plantas industriais em regiões estratégicas e pelo aumento significativo do volume de celulose produzido e exportado. A expansão da produção de celulose no país se deve a uma diversidade de fatores e processos intensificados a partir da década de 1990 em face do avanço da globalização. Dentre eles, destacam-se: grande disponibilidade de terras e mão de obra barata, boas condições edafoclimáticas que, somadas a um elevado nível tecnológico, resultam na alta produtividade florestal, e o investimento de capitais estrangeiros no setor (MARQUES, 2015).

A maior parte da celulose produzida no Brasil destinase à para exportação, cerca de $67 \%$ atualmente. Contudo, o papel aqui produzido, em sua maioria, é consumido no mercado interno, sendo os excedentes comercializados no mercado externo (IBÁ, 2018).

Apesar do avanço tecnológico e das transformações digitais, o consumo de papel em nível mundial está em franca expansão, ultrapassando recentemente 400 milhões t. Mais da metade desse consumo ocorrem na China, EUA e Japão, somada a mais um quarto na Europa. A média global é de $55 \mathrm{~kg}$ por pessoa por ano. Embora o consumo de papel esteja diminuindo ligeiramente na América do Norte, está aumentando notavelmente na Ásia e impulsionando a expansão industrial (FAO, 2019).

Enquanto a América do Norte (EUA e Canadá) em 2015 (último relato da FAO) ainda é o maior produtor de celulose, seguido pela Ásia, Europa, América Latina e Rússia, percebe-se um deslocamento da capacidade instalada principalmente para a América do Sul (sobretudo ao Brasil), Rússia, Ásia (principalmente Indonésia) e norte da Europa (MARTIN \& HAGGITH, 2018).

É fundamental que o Brasil detenha informações atualizadas e fidedignas sobre sua indústria, notadamente o setor de papel e celulose que possui posição de destaque. A FAO (Food and Agriculture Organization of the United Nations, Organização das Nações Unidas para Alimentação e Agricultura), alimenta continuamente o seu banco de dados de estatísticas de produtos florestais denominado FAOSTAT (FAO, 2019).

Os dados brutos são providos pelo governo brasileiro por meio do Sistema de Informações Florestais, em um processo de esforço contínuo de aperfeiçoamento das estatísticas florestais do país (SANQUETTA et al., 2018a). Esse banco de dados contempla registros anuais de produção, exportação e importação desde 1961.

Apesar destes dados serem públicos, eles foram pouco explorados cientificamente. Há lacunas na literatura a respeito da dinâmica temporal produção e comercialização de papéis pelo Brasil. Por conseguinte, este estudo teve como objetivo analisar a produção, importação, exportação e consumo aparente de três principais tipos de papéis produzidos no país em um período de 55 anos (1961 a 2016), desde que foi iniciada a série produzida pela FAO.

\section{MATERIAL E MÉTODOS}

Os dados utilizados neste estudo foram obtidos diretamente do site da FAO, no sistema FAOSTAT (FAO, 2019), contendo registros de produção, importação e exportação de vários tipos de papel. Este trabalho limitouse à análise de três tipos de papéis:

- W+P: papéis de escrever e imprimir (writing and printing papers);

- $\quad \mathrm{H}+\mathrm{S}$ : papéis de uso doméstico e sanitário (household and sanitary papers); e

- $\quad \mathrm{N}$ : papel jornal (newspaper).

Para cálculo do consumo aparente anual de 1961 a 2016, foi utilizada a Equação 1, que expressa a diferença entre a soma da produção e da importação menos a exportação:

$$
C A(i)=P(i)+I(i)-E(i)
$$

Em que: $C A(i)=$ consumo aparente dos papéis no ano $i[\mathrm{t}] ; P(i)=$ produção no ano $i[\mathrm{t}] ; E(i)=$ exportação no ano $i[\mathrm{t}] ;$ e $I(i)=$ importação no ano $i[\mathrm{t}]$. 
Para o cálculo do consumo anual per capita (Equação 2) foram utilizados dados populacionais do IBGE (IBGE, 2018):

$$
\operatorname{CApc}(i)=\left(\frac{C A(i)}{\operatorname{Pop}(i)}\right) / 1000
$$

Em que: $C A p c(i)=$ consumo aparente per capita dos diferentes tipos de papel no ano $i\left[\mathrm{~kg} \cdot \mathrm{hab}^{-1}\right]$; e $\operatorname{Pop}(i)=$ população do Brasil no ano $i$ [no de habitantes].

\section{RESULTADOS}

\section{Produção de papel}

No período analisado foram produzidas $109 \mathrm{M}$ t dos três tipos de papéis, sendo $77 \mathrm{M}$ t de $\mathrm{P}+\mathrm{W}, 24 \mathrm{M}$ t de $\mathrm{H}+\mathrm{S}$ e $9 \mathrm{M}$ t de $\mathrm{N}$, o que representa em termos percentuais $70 \%$ $22 \%$ e $8 \%$, respectivamente. A produção média anual desses papéis no período é de cerca de $2 \mathrm{Mt}$.

A produção de $\mathrm{P}+\mathrm{W}$ seguiu um curso de aumento até 2010, quando atingiu o seu pico de 2,7 M t, iniciando-se após esse período uma inversão, com queda gradual da fabricação desse produto pelas indústrias (Figura 1).

Por sua vez, a produção de $\mathrm{H}+\mathrm{S}$ vem apresentando crescimento contínuo, indicando que há uma tendência de expansão por parte das indústrias nesse tipo de papel.

Já a produção de $\mathrm{N}$ revela comportamento evidente de declínio, tendo atingido o ponto máximo nas décadas de 1980 e 2000 e desde então a tendência foi de queda. Atualmente (base 2016), papéis $P+W$ representam 67\% da produção, $\mathrm{H}+\mathrm{S}$ representam $31 \%$ e $\mathrm{N}$ apenas $2 \%$.

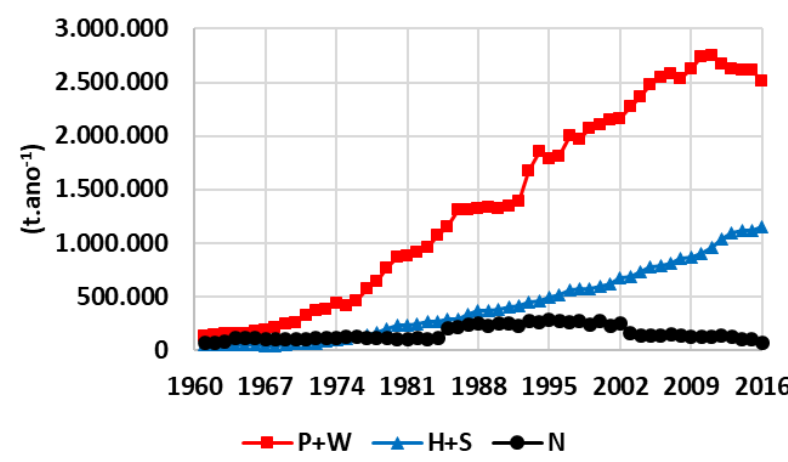

Figura 1. Produção de papéis no Brasil entre 1961 e 2016 $\mathrm{P}+\mathrm{W}$ : papéis de imprimir e escrever, $\mathrm{H}+\mathrm{S}$ : papéis de uso doméstico e sanitário, $\mathrm{N}$ : papel jornal.

Fonte: adaptado de FAO (2019).

\section{Importação de papel}

A importação total dos três tipos de papéis no período de 55 anos foi de $22 \mathrm{M} \mathrm{t}$, com média anual de $406 \mathrm{mil} \mathrm{t}$. Desse total, $10 \mathrm{M}$ t foram de $\mathrm{P}+\mathrm{W}, 188 \mathrm{mil}$ t de $\mathrm{H}+\mathrm{S}$ e $12 \mathrm{M}$ t de $\mathrm{N}$, representando em termos relativos $44 \% 1 \%$ e $55 \%$, respectivamente.

Esses valores se alteraram bastante ao longo da série temporal e, em 2016, a proporção de importação foi de $70 \% 4 \%$ e $27 \%$, respectivamente para $\mathrm{P}+\mathrm{W}, \mathrm{H}+\mathrm{S}$ e N (Figura 2). Em outras palavras, houve um crescimento mais expressivo da importação de $\mathrm{P}+\mathrm{W}$ em detrimento de $\mathrm{N}$.

Já a importação de $\mathrm{H}+\mathrm{S}$, embora tenha apresentado crescimento, não atingiu valores expressivos. Observou-se um decréscimo da importação de P+W e N a partir de 2010, o que não aconteceu com $\mathrm{H}+\mathrm{S}$.

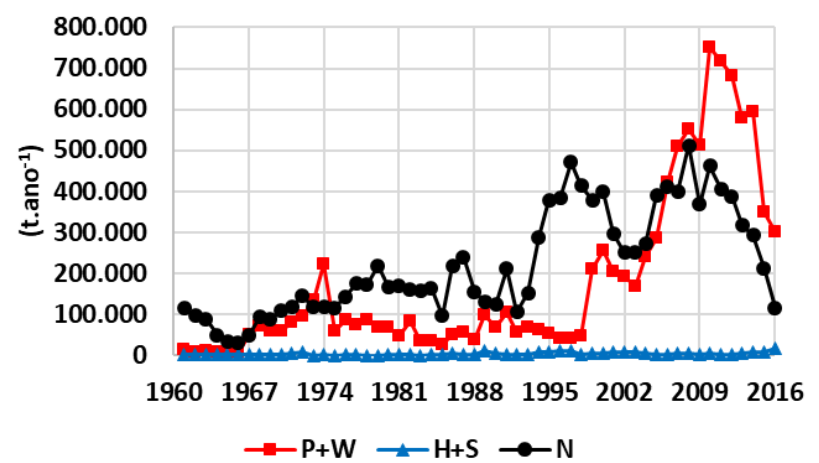

Figura 2. Importação de papéis no Brasil entre 1961 e 2016 $\mathrm{P}+\mathrm{W}$ : papéis de imprimir e escrever, $\mathrm{H}+\mathrm{S}$ : papéis de uso doméstico e sanitário, $\mathrm{N}$ : papel jornal.

Fonte: adaptado de FAO (2019).

\section{Exportação de papel}

Foram exportadas $26 \mathrm{M}$ t de papéis no período considerado, com média de 478 mil t anuais. Portanto, a exportação supera a importação, resultando num superávit na balança comercial.

A maior parte das exportações foi de $\mathrm{P}+\mathrm{W}$, com $97 \%$ do volume no período completo e $99 \%$ em 2016 . O percentual de participação nas exportações dos outros papéis é pouco relevante (Figura 3 ).

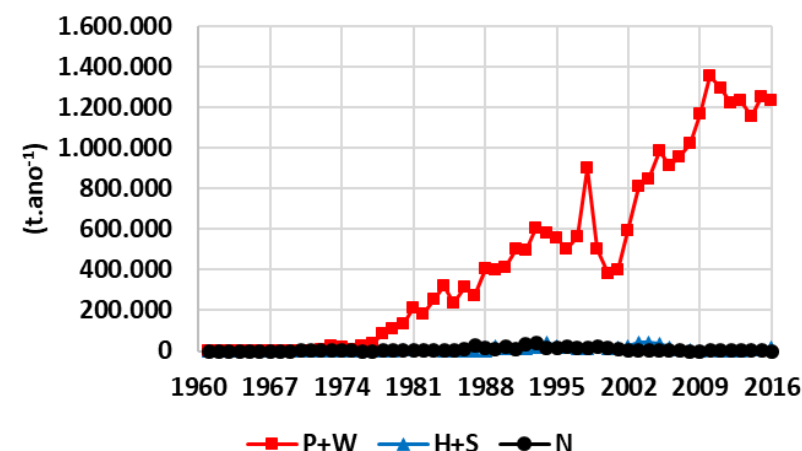

Figura 3. Exportação de papéis no Brasil entre 1961 e 2016 $\mathrm{P}+\mathrm{W}$ : papéis de imprimir e escrever, $\mathrm{H}+\mathrm{S}$ : papéis de uso doméstico e sanitário, $\mathrm{N}$ : papel jornal.

Fonte: adaptado de FAO (2019). 
Observa-se uma trajetória de crescimento das exportações até 2010, quando se inicia, a partir de então, um período de decréscimo. Picos de exportação foram observados em alguns anos, como em 1998 e posteriormente em 2010, quando foi atingido o volume máximo em toda a série de 1,4 $\mathrm{M} \mathrm{t}$.

\section{Consumo aparente de papel}

$\mathrm{O}$ consumo aparente total no período foi de $106 \mathrm{M} \mathrm{t}$, sendo $61 \mathrm{M}$ t de $\mathrm{P}+\mathrm{W}, 24 \mathrm{M}$ t de $\mathrm{H}+\mathrm{S}$ e $21 \mathrm{M}$ t de $\mathrm{N}$. A média anual de consumo é de $2 \mathrm{M}$ t. No período de 55 anos o consumo aparente teve a seguinte participação: $58 \%$ para $\mathrm{P}+\mathrm{W}, 22 \%$ para $\mathrm{H}+\mathrm{S}$ e $20 \%$ para N. Em 2016 essas razões foram de $54 \%$, $40 \%$ e $6 \%$, respectivamente, para $\mathrm{P}+\mathrm{W}, \mathrm{H}+\mathrm{S}$ e N (Figura 4).

Observou-se que houve crescimento do consumo aparente no período, mas os padrões são distintos para os três tipos de papéis. Para $\mathrm{H}+\mathrm{S}$ verifica-se um crescimento constante, enquanto para $\mathrm{P}+\mathrm{W}$ e $\mathrm{N}$ evidencia-se uma redução do consumo, principalmente a partir de 2010.

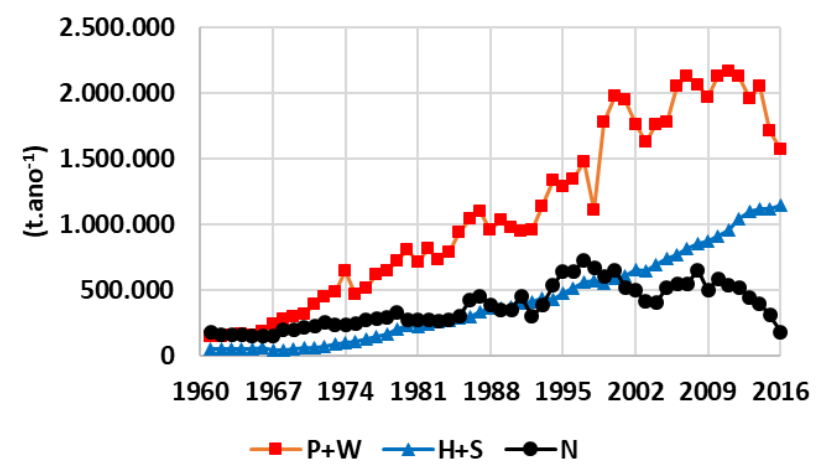

Figura 4. Consumo aparente de papéis no Brasil entre $1961 \mathrm{e}$ 2016; P+W: papéis de imprimir e escrever, $\mathrm{H}+\mathrm{S}$ : papéis de uso doméstico e sanitário, $\mathrm{N}$ : papel jornal.

A população do Brasil variou de $73 \mathrm{M}$ de habitantes em 1961 a 204 M em 2016, seguindo uma tendência aproximadamente linear. O consumo per capita dos três tipos de papéis em foco comportou-se de modo crescente até 2010 no caso de $\mathrm{P}+\mathrm{W}$ e $\mathrm{N}$, decrescendo a posteriori, e continuamente crescente para $\mathrm{H}+\mathrm{S}$.

O consumo per capita desses papéis saltou de cerca de 5 kg.hab ${ }^{-1}$.ano-1 em 1961 para 20 kg.hab ${ }^{-1}$.ano-1 em 2016, ou seja, um aumento de 4 vezes. Contudo, tem-se observado uma queda desse consumo a partir de 2010, acompanhando a produção. Isso pode estar ligado ao fraco desempenho de economia do país e às condições desfavoráveis de emprego e renda, com impactos no poder de compra dos consumidores.

\section{DISCUSSÃO}

O Brasil é o sexto maior produtor de papel do mundo, perdendo apenas para a China, Estados Unidos, Japão, Alemanha e Coreia do Sul (FAO, 2019). A produção total mundial é de 400 milhões de toneladas e cerca de um quarto desse montante é produzido na China.

O Brasil produz atualmente cerca de 10 milhões de toneladas anuais. De 2004 a 2014 o crescimento médio na produção de papéis foi de $3 \%$ ao ano. Com o crescimento da produção houve uma expansão da base florestal do país (SANQUETTA et al., 2018b).

Especificamente sobre os três tipos de papéis aqui analisados, considerando a produção de $10 \mathrm{M} \mathrm{t}$ como referência, eles representam aproximadamente $37 \%$ desse montante. Portanto, esses papéis ocupam posição de destaque na produção da indústria nacional juntamente com os papéis para embalagens, papel cartão, entre outros.

Todavia, é importante destacar as distintas curvas temporais de produção dos três tipos de papéis analisados. Enquanto $\mathrm{P}+\mathrm{W}$ e $\mathrm{N}$ apresentam tendência de declínio, $\mathrm{H}+\mathrm{S}$, ao contrário, mostram perspectivas de expansão. Isso quer dizer que a indústria está passando a direcionar a sua produção a outros tipos de papéis nos últimos anos, acompanhando as tendências de consumo.

As importações brasileiras desses papéis, sobretudo $\mathrm{P}+\mathrm{W}$ e $\mathrm{N}$, caíram muito a partir de 2010 e se encontram atualmente próximas dos patamares de décadas de 1990 . O Brasil tem tradicionalmente importado apenas pequenas quantidades de $\mathrm{H}+\mathrm{S}$. Essa queda nas importações tem relação com a performance ruim da economia brasileira e a crise instalada nesse período. Por sua vez, essa crise tem diversas causas e consequências, conforme reportado por BARBOSA-FILHO (2017).

As exportações de $\mathrm{H}+\mathrm{S}$ e $\mathrm{N}$ são pouco expressivas na série temporal em comparação àquelas de $\mathrm{P}+\mathrm{W}$, onde mais de $99 \%$ do volume exportado é desse tipo de papel. A queda nas exportações experimentada desde 2010, combinada com a crise econômica que repercutiu no consumo interno, induziram a diminuição da produção desses tipos de papéis.

Essa queda nas exportações decorre da tendência de redução de consumo desse tipo de papel em vários países. Atualmente um tema em evidência é o "papel versus digital". Muitas aplicações gráficas e de escrita, desde livros, revistas, contas e relatórios corporativos, estão mudando de cópia impressa em papel para formulário 
eletrônico (MARTIN \& HAGGITH, 2018).

$A$ redução no consumo aparente doméstico de $\mathrm{P}+\mathrm{W}$ e $\mathrm{N}$ também é evidente. Num paralelo com outras nações o Brasil se alinha para reduzir o consumo de papéis gráficos (RISI, 2016). São grandes os esforços na atualidade para usar menos esse tipo de papel, seja por empresas, indivíduos e governos.

Todos vêm investindo em mais equipamentos de informática e programas computacionais, treinamento de funcionários e campanhas de conscientização. Alguns até sonham com uma sociedade livre de papel, embora o produto esteja presente em toda a vida humana e seu consumo tenha crescido em $400 \%$ nos últimos 40 anos (SHENOY \& AYTHAL, 2016). Apesar da queda no consumo, o papel do tipo $\mathrm{P}+\mathrm{W}$ é um dos papéis de maior relevância econômica no Brasil (SILVA et al., 2015).

Ficou evidente que há uma expansão da produção e consumo doméstico de papel $(\mathrm{H}+\mathrm{S})$, também chamados de papéis tissue, que são os usados em higiene pessoal e limpeza doméstica, tais como papel higiênico, papeltoalha, guardanapos, fraldas descartáveis, absorventes, entre outros (COSTA \& BACHA, 2017). A indústria está direcionando seus esforços para atender a demanda do consumidor e localizar os melhores nichos de mercado.

O consumo per capita desses papéis teve grande crescimento desde 1961, praticamente quadruplicando. Apesar da queda geral no consumo per capita do conjunto desses três papéis, há uma tendência consistente de aumento do consumo de $\mathrm{H}+\mathrm{S}$ mesmo com a grave crise econômica que se abateu sobre o país. Há uma tendência de crescimento do consumo de $\mathrm{H}+\mathrm{S}$ no mundo (RISI, 2016), decorrente, em grande parte, do consumo desses produtos na Ásia. Embora tenha apresentado queda, $\mathrm{P}+\mathrm{W}$ tem um padrão de consumo superior aos demais.

\section{CONCLUSÕES}

Em 55 anos houve expressivo crescimento da produção de papéis no Brasil pela indústria, o que veio acompanhado de aumento nas exportações e importações.

Atualmente há uma retração na produção, exportação e importação de papéis, fato decorrente, dentre outros motivos, da má performance da economia do país.

Produção e consumo de papéis de uso sanitário e doméstico $(\mathrm{H}+\mathrm{S})$ apresentam tendência de crescimento, enquanto de papéis de imprimir e de escrever $(P+W)$, bem como papéis jornal $(\mathrm{N})$, mostram trajetória de queda a partir de 2010.

O consumo per capita de papéis no Brasil quadruplicou em 55 anos, fato decorrente da consolidação desses produtos e melhoria na qualidade de vida das pessoas.

O consumo per capita de papel $\mathrm{H}+\mathrm{S}$ está em alta e é muito provável que essa tendência seja preservada nos próximos anos.

\section{AGRADECIMENTOS}

À CAPES pelo suporte financeiro a este trabalho, por meio de bolsa de Pesquisador Estágio Sênior de PósDoutorado no Exterior ao primeiro autor junto à Universidade de Lisboa, Portugal.

\section{REFERÊNCIAS}

BARBOSA-FILHO, F.H. A crise econômica de 2014/2017. Estudos Avançados, v.31, n.89, p.51-60, 2017.

COSTA, L.A.; BACHA, C.J.C. Análise da estrutura produtiva e do consumo de papéis tissue no Brasil. Teoria e Evidência Econômica, v.23, n.48, p.118-149, 2017.

FAO - Food and Agriculture Organization of the United Nations. FAOSTAT. 2017. Disponível em: http://www.fao.org/faostat/ en/\#data/GF

IBÁ - INSTITUTO BRASILEIRO DE ÁRVORES. Relatório 2017. São Paulo: IBÁ, 2018. Disponível em: https://iba.org/images/shared/ Biblioteca/IBA_RelatorioAnual2017.pdf

IBÁ - INSTITUTO BRASILEIRO DE ÁRVORES. Sumário executivo 2018. São Paulo: IBÁ, 2018. Disponível em: https://iba. org/datafiles/publicacoes/relatorios/digital-sumarioexecutivo2018.pdf

IBGE - Instituto Brasileiro e Geografia e Estatística. Estimativas da População. 2018. Disponível em: https://www.ibge.gov.br/ estatisticas-novoportal/sociais/populacao/9103-estimativas-depopulacao.html?=\&t=o-que-e

MARQUES, M.I.M. Considerações sobre a expansão da indústria de papel e celulose no Brasil a partir do caso da Suzano Papel e Celulose. GEOgraphia, n.17, v.35, p.120-147, 2015.

MARTIN, J.; HAGGITH, M. The state of the global paper industry: shifting seas: new challenges and opportunities for forests, people and climate. Environmental Paper Network, 2018. 89p.

MOREIRA, J.M.M.A.P.; OLIVEIRA, E.B. Importância do setor florestal brasileiro com ênfase nas plantações florestais comerciais. Plantações florestais: geração de benefícios com baixo impacto ambiental. Brasília: Embrapa, 2017.

RISI - RESOURCE INFORMATION SYSTEMS INC. Annual Review of Global Pulp and Paper Statistics. ICFPA (International Council of Forest \& Paper Associations) Sustainability Progress Report. 2016. Disponível em: https://www.risiinfo.com/product/annualreview-of-global-pulp-paper-statistics/

SANQUETTA, C.R. et al. Dynamics of carbon and $\mathrm{CO}_{2}$ removals by Brazilian forest plantations during 1990-2016. Carbon Balance 
and Management, v.13 n.20, p.1-12, 2018a.

SANQUETTA, C.R. et al. Dinâmica em superfície, volume, biomassa e carbono nas florestas plantadas brasileiras: 19902016. BIOFIX Scientific Journal, v.3, n.1 p.152-160, 2018b.

SHENOY, P.A; AYTHAL, P.S. Study on History of Paper and possible Paper Free World. International Journal of Management, IT and Engineering, v.6, n.1, p.337-355. 2016.

SILVA, D.A.I. et al. Life cycle assessment of offset paper production in Brazil: hotspots and cleaner production alternatives. Journal of Cleaner Production, v.93, p.222-233. 2015. 